Original article

\title{
Satisfactory outcomes after orthognathic surgery with surgically assisted rapid maxillary expansion using a hybrid device
}

\author{
Helen Baranto ${ }^{1)}$, Carina K. Weiner ${ }^{1)}$, Idil A. Burt ${ }^{2)}$, and Annika Rosén ${ }^{3)}$ \\ 1) Department of Oral and Maxillofacial Surgery, Folktandvården Eastman Institutet, Stockholm, Sweden \\ ${ }^{2}$ Division of Orthodontics, Department of Dental Medicine, Karolinska Institute, Huddinge, Sweden \\ ${ }^{3}$ Department of Clinical Dentistry, Section of Oral \& Maxillofacial Surgery, Faculty of Medicine and Dentistry, University of Bergen, Bergen, Norway
}

(Received February 20, 2019; Accepted July 11, 2019)

\begin{abstract}
The self-reported functional outcomes, clinical findings, and results of dental cast analysis before and after orthognathic surgery with surgically assisted rapid maxillary expansion (SARME) using a hybrid rapid maxillary expander (RME) were evaluated. Data were collected from 43 patients who underwent orthognathic surgery with SARME using a hybrid RME between 2001 and 2013. The patients were recruited during a follow-up clinical examination and were required to complete a questionnaire about their opinions and self-reported functional outcomes. Dental casts were used to analyze posttreatment palatal expansion. The mean follow-up time was 68 months (range: $25-135$ months). The most common indication for SARME was the presence of a crossbite. Of the 30 patients who underwent a follow-up clinical examination (69.8\% answer rate), $4(13.3 \%)$ had symptoms of temporomandibular disorder (TMD), $1(3.3 \%)$ experienced myalgia, and $3(10.0 \%)$ experienced arthralgia on clinical palpation. Cast analysis revealed significant palatal expansion. The intercanine distance, intermolar distance, and palatal height were increased by 3,5 , and $2 \mathrm{~mm}$, respectively. Overall, the patients were satisfied with the preoperative information, improved functions, and aesthetic results. The prevalence of TMD symptoms and other side effects following orthognathic surgery with SARME using a hybrid RME was low, and significant palatal expansions were achieved.
\end{abstract}

Keywords; hybrid RME device, orthognathic surgery, palatal expansion technique, surgically assisted rapid maxillary expansion, temporomandibular disorder, treatment outcomes and satisfaction

\section{Introduction}

Growth disturbances in the maxilla can lead to crowding of the teeth and a posterior crossbite, which can, in turn, cause nasal obstruction and oral breathing $[1,2]$. Orthognathic surgery with surgically assisted rapid maxillary expansion (SARME) can improve facial-skeletal relationships and dental occlusion. Previous studies have shown that patients seek surgical orthodontic treatment to improve their facial and dental appearance and to correct functional occlusion discrepancies [3-5]. Although orthognathic surgery can yield functional and psychosocial improvements [6], patients should be informed about the duration and side effects of these treatments $[5,7,8]$.

SARME can correct transverse maxillomandibular discrepancies in skeletally mature patients from the age of 13 [9-11]. Both unilateral and bilateral transverse hypoplasia in skeletally mature patients can be corrected to provide dental arch space and to enlarge the maxillary apical base and palatal vault. The tongue is the most important source of pressure and is in direct contact with both the dental arches and the jaws; therefore, the size, posture, and function of the tongue are of utmost importance [12].

Correspondence to Dr. Helen Baranto, Department of Oral and Maxillofacial Surgery, Folktandvården Eastman Institutet, Dalagatan 11 box 6031, Stockholm 10231, Sweden E-mail: helen.baranto@sll.se

Color figures can be viewed in the online issue at J-STAGE.

doi.org/10.2334/josnusd.19-0078

DN/JST.JSTAGE/josnusd/19-0078
SARME creates more space for the tongue, thereby assisting the swallowing process and preventing relapse [6].

Studies have shown that tooth- or bone-borne devices can be successfully used to expand a narrow maxilla by SARME $[13,14]$. However, these expander devices also present with some disadvantages and complications $[15,16]$. A few studies on hybrid rapid maxillary expansion (RME) devices have been published in the literature; nonetheless, the popularity of these devices is increasing owing to their relatively low rates of failure [17] and risk of tooth damage because the force is primarily applied to the bone [18]. Consequently, a hybrid RME device was developed at the Department of Oral and Maxillofacial Surgery, Karolinska Institute, Sweden. A modified hyrax appliance with both tooth and bone anchorage was used. The treatment effects of this appliance have not been evaluated so far.

According to a systematic review, it is not possible to establish an association among posterior crossbite, muscle pain, and disc displacement because equal number of studies both support and refute the associations [19]. Thus, studies regarding malocclusion and temporomandibular disorder (TMD) symptoms have not produced consistent results. Some studies report improvement of TMD symptoms after orthognathic surgery, whereas others show no improvement or worsening of the temporomandibular joint (TMJ) functions [20-22]. The other risks following orthognathic surgery include nerve injury with sensory disturbances in the soft tissue [23].

It is also notable that these patients often present with severe malocclusions that frequently require a second surgery following SARME to correct jaw relation [15]. Additionally, uni- or bimaxillary surgery is conducted to correct the occlusion if required [24].

This study aimed to examine the self-reported functional outcomes of SARME and the results of a dental cast analysis before and after orthognathic surgery using a hybrid RME device. The hypotheses tested in this study were as follows: 1) a normal occlusion with significant palatal expansion is achievable following orthognathic surgery with SARME using a hybrid RME device, and 2) patients are satisfied with the treatment results and the low prevalence of side effects.

\section{Materials and Methods}

Using a descriptive research methodology, this three-part study evaluated the outcomes of orthognathic surgery with SARME utilizing a hybrid device. In Part I, data were retrospectively collected from the medical records of the patients. In Part II, clinical findings were evaluated in correlation with the patients' self-reported functional outcomes. In Part III, palatal expansion was analyzed using the study casts. Figure 1 illustrates the design of the study.

The study participants included a cohort of consecutive patients $(n=$ 43) aged between 13 and 50 years who underwent orthognathic surgery with SARME between 2001 and 2013 at the Department of Oral and Maxillofacial Surgery, Karolinska Institute, Sweden. The 43 patients were referred to the department by nine different orthodontic specialists from the Public Dental Services of Stockholm County Council or from private clinics. The patients were selected for surgery after meeting with oral and maxillofacial surgeons and orthodontists.

All patients were treated for narrow maxillas with posterior crossbite and prescribed a modified hyrax tooth- and bone-anchored expander device (Fig. 2), which was fabricated by dental technicians at the Karo- 


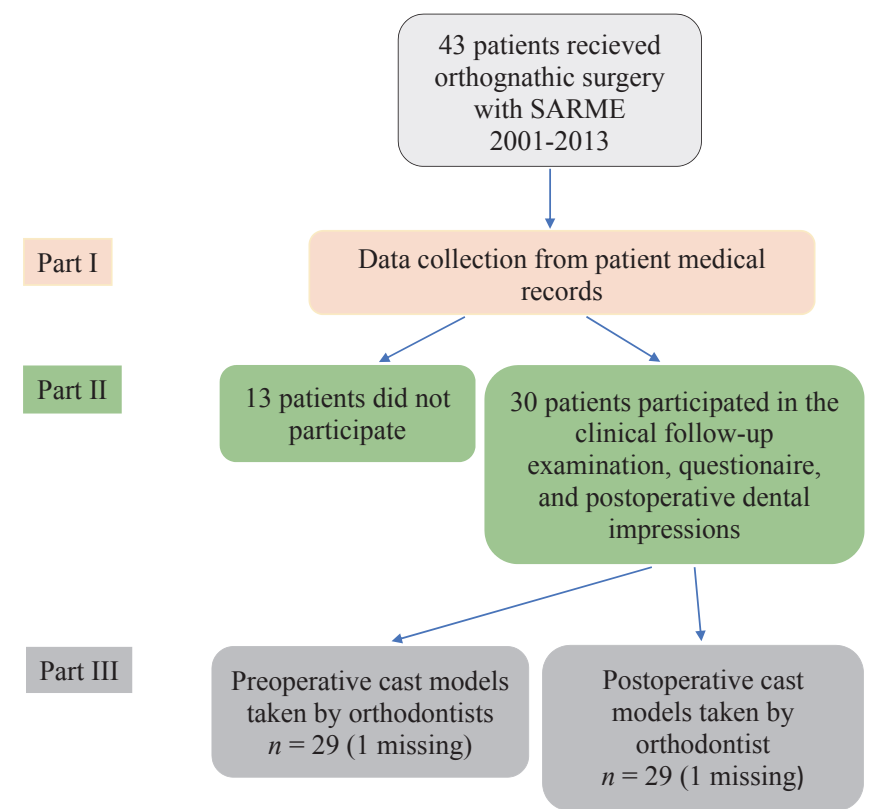

Fig. 1 Flow Chart of Study Design. Data were collected from the medical records of the patients (Part I), clinical follow-up examinations and questionnaires (Part II), and pre- and postoperative cast model analyses (Part III)

linska Institute. This device has a low risk of tooth damage [18] and is tooth- and bone-anchored, which aids in the redirection of the force from the teeth to the bone. The minimum follow-up period in this study was 6 months. Patients with craniofacial anomalies and those who did not meet the previously mentioned inclusion criteria were excluded. The approval for this study was obtained from the ethical committee in Stockholm, Sweden (DNR: 2012/4:3).

All patients were preoperatively and clinically examined by the surgeon before treatment with SARME orthognathic surgery. At this time, the occlusion, TMJ status, and myofascial status were assessed and registered in the patients' records. SARME treatment was initiated with the installation of two 5-mm titanium bone screws (KLS Martin, Tuttlingen, Germany) into the palate, one on each side of the midline, close to the molars. Alginate impressions were taken, and the hybrid tooth- and bone-anchored RME device (see Fig. 2) was installed 1 to 2 days before surgery. Under general anesthesia, a Le Fort I osteotomy procedure was performed with an osteotomy in the midline of the maxilla without pterygomaxillary disjunction. The midline osteotomy enabled the expansion of the maxilla during the distraction phase, which was tested during the surgical procedure.

Approximately 1 week after surgery, the surgeon instructed the patient to set the expansion device to 0.25 to $1.00 \mathrm{~mm}$ per day. The distraction lasted for approximately 14 days or until the expansion was increased enough to visualize an open space between the central incisors (up to 15 $\mathrm{mm}$ ). The distraction device remained attached to the teeth and palate for approximately 4 to 6 months (retention period) to maintain palatal expansion and to allow the bone to heal. During retention, the orthodontist bonded MBT.022 system braces (3M Unitek, St. Paul, MN, USA) to the teeth in the upper and lower jaws to achieve consistent dental arches. This treatment lasted for approximately 1 year. After the orthodontic treatment was completed, bonded retainers made of stainless-steel coaxial wires (MASEL Penta-One coaxial straight length 14."0215) were used as retention appliances in the upper and lower anterior regions to prevent relapse.

The patients were examined by an orthodontist and a surgeon 1 year after the SARME treatment to determine whether they needed a second surgery to correct the jaw relation. Uni- or bi-maxillary surgery was conducted to correct the teeth occlusion using Le Fort I osteotomy in the maxilla, intraoral vertical ramus osteotomy (IVRO), segmental ramus osteotomy (SRO), or bilateral sagittal split osteotomy (BSSO) in the mandible if required.

\section{Part I: data collection from patient records}

The patients' records were reviewed at the Department of Oral and Maxillofacial Surgery using the TakeCare digital medical record system

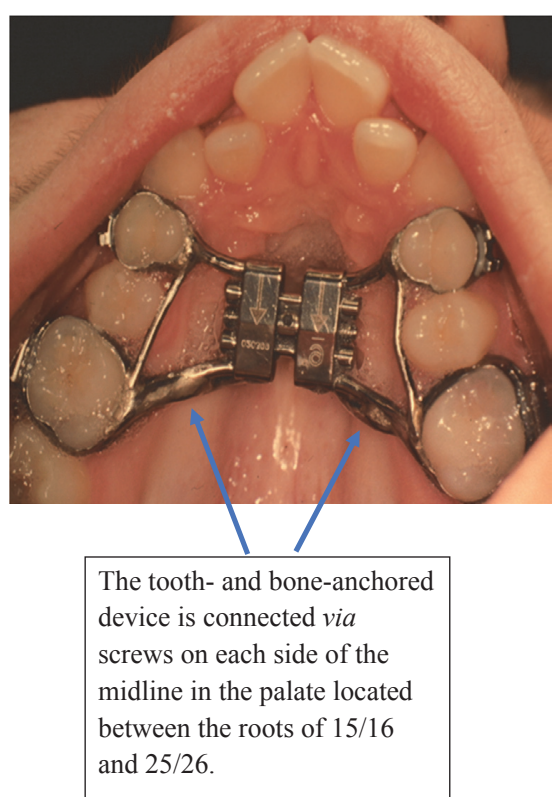

Fig. 2 The modified Hyrax tooth- and bone- anchored device. The modified Hyrax tooth- and bone-anchored expander device was attached to the teeth and palatal bone by inserting palatal screws on each side. The screws are hidden under the posterior part of the device.

(CompuGroup Medical). The preoperative data included gender, age, dental occlusion, and intra- and extra-oral conditions, whereas the postoperative data included the duration of orthognathic treatment, type of surgery or surgeries, and dental occlusion. All collected data were password-protected and coded before incorporation into a Microsoft Excel document.

\section{Part II: clinical follow-up examination and the questionnaire}

The patients $(n=43)$ were contacted by telephone to request for voluntary participation in the study; among them, 30 patients $(69.8 \%$; 12 women and 18 men) agreed to participate, whereas $13(30.2 \%)$ declined to participate in the follow-up examination owing to relocation $(n=8 ; 61.5 \%)$ and lack of interest ( $n=5 ; 38.5 \%$; Fig. 1). A general dental practitioner who did not participate in the treatment planning or execution conducted all the clinical examinations and distributed the questionnaire.

The clinical follow-up included an evaluation of the occlusion and TMJ status, including myofacial muscles and sensory disturbances in the oral mucosa and lips. The sagittal, vertical, and transversal relations of the mandible were assessed for dental occlusion according to Angle's classification [W.R. Proffit et al, Contemporary Orthodontics, 5th ed., 204-214, Mosby Elsevier Inc. (ISBN 978-0-323-08317-1), 2012]. TMJ function was evaluated according to a simplified diagnostic criteria for TMD; by measuring the maximum opening capacity between the incisors to calculate the laterotrusion, mediotrusion, and protrusion in $\mathrm{mm}$; and by assessing the presence of clicking or crepitation sounds from the joints. Unlimited, unassisted opening capacities of $\geq 35 \mathrm{~mm}$ were classified as normal. Normal, unlimited laterotrusion, mediotrusion, and protrusion were established as $\geq 7 \mathrm{~mm}$. Muscle tenderness was measured by palpation on a scale of 0 to 3 , with 0 indicating no pain, 1 representing mild pain, 2 indicating moderate pain, and 3 indicating severe pain [25]. The lips and gingiva were examined for changes in sensory perception (e.g., hypo- or hypersensitivity) using a simplified quantitative sensory pinprick test with a dental probe and toothbrush [26]. Each patient served as their own control, wherein untreated areas of the palatal and lingual gingiva with no incisions were used as references. The questionnaire comprised 10 questions regarding the patients' opinions about their treatment outcomes (TMJ status, aesthetic appearance, and sensory disturbances in the oral mucosa or lips). Alginate impressions of the jaws were taken at the follow-up examination for comparison with preoperative casts.

\section{Part III: cast analysis}

Twenty-nine preoperative casts were collected from the orthodontic clinics; the remaining preoperative casts were missing because the clinics did not save them after a period of 10 years. Postoperative dental casts $(n$ 


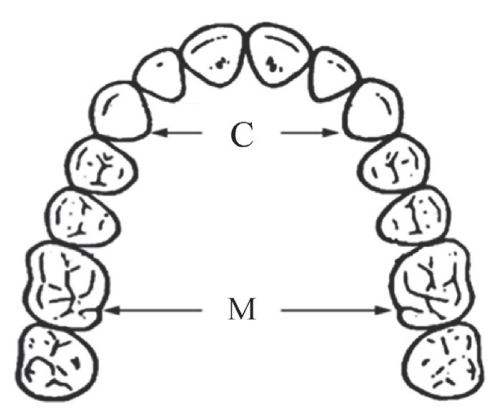

Magnusson et al. 2009

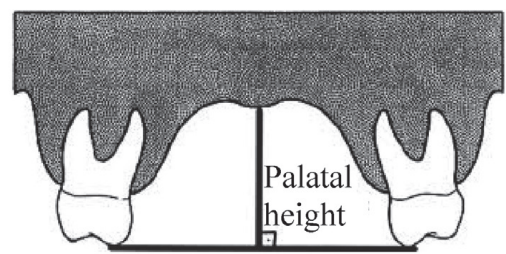

Sokucua et al. 2009

Fig. 3 Measurements of the pre- and postoperative cast models. (a) measurements of the pre- and postoperative cast models were performed using a sliding vernier caliper. Intermaxillary measurements were taken at two anterior and two posterior reference points. $\mathrm{C}$ denotes the intercanine width, the distance between the most prominent cervical points of the palatal ridge on the canines (two anterior reference points). $\mathrm{M}$ denotes the intermolar width, the distance between the most cervical points of the palatal fissure on the first molars (two posterior reference points). (b) palatal depth was measured as the distance from the mid-deepest part of the palate to the line connecting the left and right disto-lingual cusp tips of the upper first molars.

= 29) were produced at follow-up examinations. Pre- and postoperative casts were used to analyze the palatal expansion and changes in dental occlusion. A vernier caliper device was used to measure intercanine and intermolar widths. Additionally, the palatal heights were measured (Fig. 3). Subsequently, the casts were collected, produced, and analyzed by the same general dental practitioner who conducted the follow-up examinations.

\section{Statistical analysis}

All collected data were compiled and statistically analyzed using Microsoft Excel and the statistical program Statistica (TIBCO Software Inc., Palo Alto, CA, USA). The Shapiro-Wilk test was used to determine if the data were normally distributed. Differences in means were calculated using the $t$-test and Mann-Whitney $U$-test. The significance was set at $P \leq 0.05$.

\section{Results}

\section{Results for part I: data collection}

A total of 43 patients ranging in age from 14 to 40 years old who received orthognathic treatment with SARME were recruited in this study. Among them, 19 were females $(44.2 \%)$ with a mean age of 19.2, and 24 were males $(55.8 \%)$ with a mean age of 19.1 . The patients presented with malocclusion (unilateral or bilateral posterior crossbite, open bite or deep bite), with the most common type being bilateral crossbite $(n=35 ; 81.4 \%)$. Some patients presented more than one type of malocclusion. Preoperatively, all patients presented a normal unassisted opening capacity along with normal laterotrusion, mediotrusion, and protrusion capacities. Two patients (4.7\%) had preoperative myalgia. No joint sounds were recorded among the 43 participants, and no sensory disturbances of the lips or oral mucosa were observed.

Further evaluations of the dental occlusions were made after orthognathic treatment with SARME. Among the 43 patients, 31 (72.1\%) continued to present with malocclusion that required a second surgery; 4 of the 31

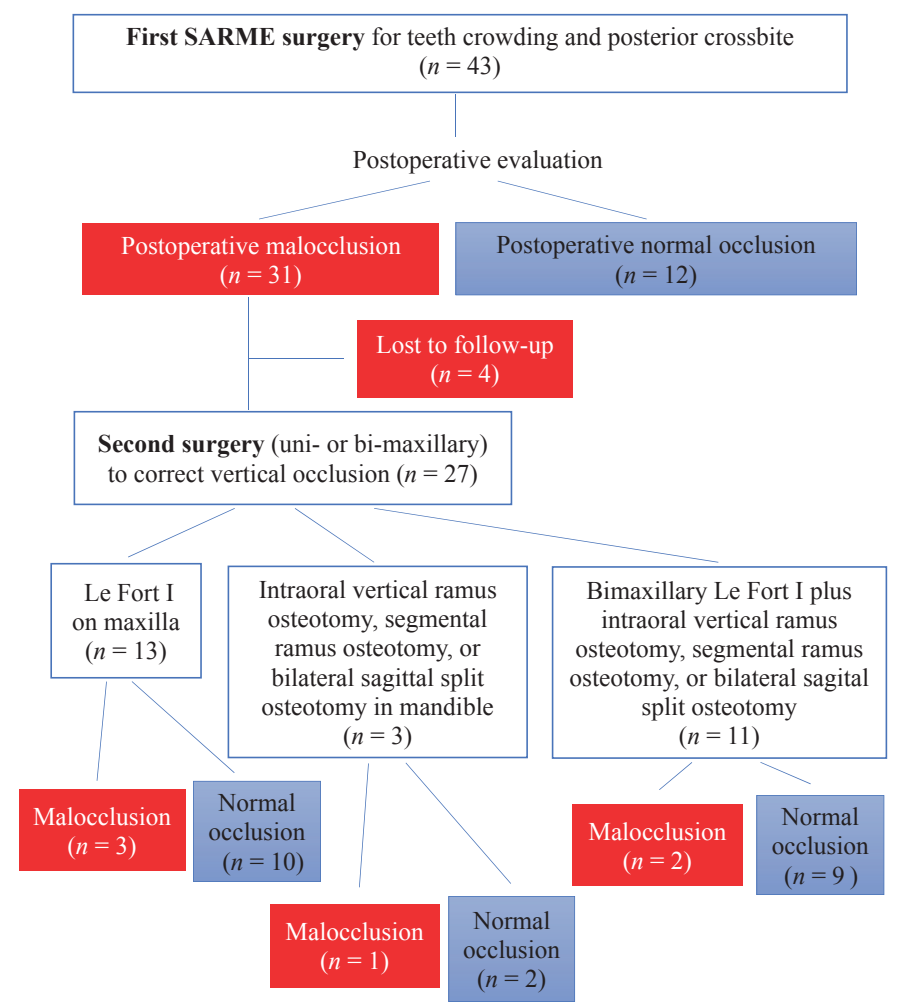

Fig. 4 Flow chart of orthognathic treatment with SARME. Among the 43 patients who received SARME treatment, 12 showed neutral occlusion, and 31 needed a second surgery due to malocclusion ( 2 declined the second surgery because they were satisfied with the treatment result, 1 declined because he or she was out of the country, and 1 did not cooperate). Of the 27 patients who received a second surgery, 21 showed neutral occlusion, and 6 continued to present with malocclusion. In total, 33 patients demonstrated neutral occlusion, whereas 10 patients continued to present with malocclusion after completing the treatment.

patients $(12.9 \%)$ did not cooperate or choose to proceed with a second surgery, whereas 27 patients $(87.1 \%)$ agreed to undergo a second surgery. Furthermore, 13 (48.1\%) of the 27 patients who agreed to undergo a second surgery received a Le Fort I osteotomy to adjust the maxilla; 3 (11.1\%) received SRO, BSSO, or IVRO to adjust the mandible; and $11(40.7 \%)$ underwent bimaxillary surgery. After treatment, 33 patients $(76.7 \%)$ presented normal postoperative sagittal, vertical, and transversal relations of occlusion, whereas 10 (23.3\%) presented malocclusion (Fig. 4).

\section{Results for part II: clinical follow-up examination and questionnaire} Clinical Assessment

The mean follow-up time was 68 months (25-135 months). Among the 30 participants in Part II, 24 (80.0\%) presented with Angle's Class I occlusion with an overbite of 1-3 $\mathrm{mm}$ and no transversal discrepancies, indicating that normal occlusion was achieved after orthognathic surgery with SARME, using a hybrid RME device. None of the patients presented impaired or reduced capacity of mandibular movement at follow-up. One patient (3.3\%) reported pain on palpation of one joint, and two patients $(6.7 \%)$ experienced tenderness in both joints. Another patient (3.3\%) complained of pain in the masseter and temporalis myofascial muscles on palpation. Nine patients $(30.0 \%)$ presented with clicking sounds in the joint, whereas crepitations were not heard in any of the patients. As mentioned in the results of Part I, two patients (4.7\%) showed preoperative myalgia with no signs of TMD at follow-up. Twenty patients $(66.7 \%)$ subjected to surgery presented with visual scar tissue in the intraoral area. Eighteen patients $(60.0 \%)$ experienced hyposensitivity in the vestibular maxillary oral mucosa, and four (13.3\%) experienced hyposensitivity in the upper lip.

\section{Questionnaire}

Of the 30 patients who completed the questionnaire, 29 (96.7\%) reported that they were satisfied with the orthognathic treatment with SARME using the hybrid RME device, and none regretted having undergone the treatment. Twenty-eight patients $(93.3 \%)$ felt that they had received suf- 


\section{Did SARME treatment alter complaints for...}

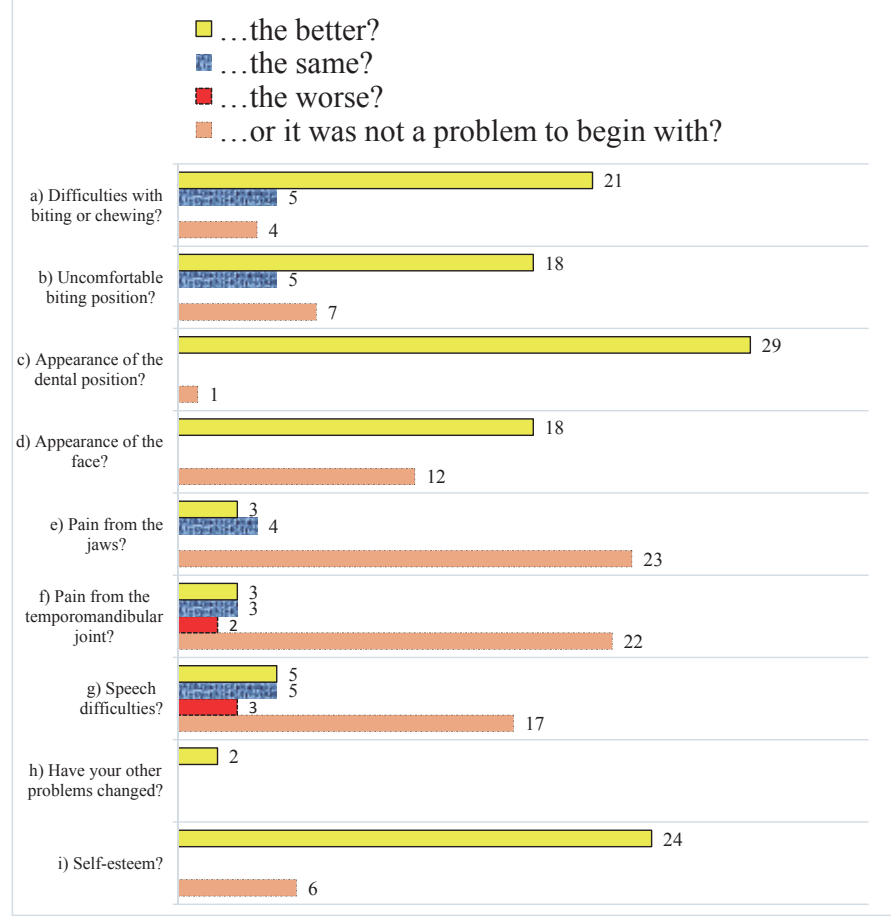

Fig. 5 Multiple-choice questionnaire about alteration of preoperative complaints due to orthognatic surgery with SARME using a hybrid device. Multiple-choice questionnaire about alteration of preoperative complaints (e.g., facial appearance, dental occlusion, temporomandibular disorders, speech, and self-esteem) due to surgery were evaluated. The discomforts of most patients were either not present from the start or had improved after orthognatic surgery with SARME using a hybrid device. Some patients observed no improvement as a result of the surgery, whereas five patients reported worsening of the preoperative speech difficulties and pain in the temporomandibular joint.

ficient preoperative information. According to most of the patients, other preoperative difficulties (such as biting and chewing, dental position, facial appearance, speech, self-esteem) had improved after orthognathic surgery with SARME (Fig. 5). Worsening of pain from TMJ was uncommon among the patients (6.7\%, Fig. 5). Permanent upper lip sensation disturbance bothered 4 patients (13.3\%), and 1 patient (3.3\%) was dissatisfied with the treatment due to impaired sensation in the upper lip.

\section{Results for part III: cast analyses}

Preoperative casts confirmed the epidemiologic data and diagnoses in the patient records from Part I. The most common malocclusion was bilateral posterior crossbite $(n=18 ; 62.1 \%)$. Analyses of the preoperative and postoperative casts revealed that orthognathic surgery with SARME and the hybrid RME device improved transverse occlusion and teeth alignment. Significant improvements were achieved in intermaxillary distance (intercanine: $P<0.005$; intermolar: $\left.P<0.05 \times 10^{4}\right)$ and palatal height $(P<0.05)$, as presented in Table 1.

\section{Discussion}

The present study evaluated the self-reported functional outcomes of orthognathic surgery with SARME using a hybrid RME device in correlation with the clinical findings and dental cast analyses. Overall, the patients were satisfied with the results, which included improvements in both function and aesthetics. TMD symptoms following treatment were uncommon, and significant palatal expansion was achieved. According to the medical records, most patients achieved postoperative Angle's Class I molar relation after treatment completion. However, most of the patients needed a second surgery to correct the vertical height and further improve the occlusion, thus confirming the general notion that malocclusion often involves multiple diagnoses and additional treatments [15,24].

There is no consensus in the literature regarding the type of distractor (tooth-borne or bone-borne) that should be used for SARME for the
Table 1 Analysis of cast models before and after orthognathic treatment with SARME

\begin{tabular}{lccccccccc}
\hline Tooth & & \multicolumn{2}{c}{$\begin{array}{c}\text { Before } \\
\text { treatment } \\
(\text { mean, mm) }\end{array}$} & SD & $n$ & $\begin{array}{c}\text { After } \\
\text { treatment } \\
(\text { mean, mm) }\end{array}$ & SD & $P$ value & $t$ value \\
\hline $\begin{array}{l}\text { Maxillary } \\
\text { canine gingival }\end{array}$ & $\mathrm{C}$ & 29 & 23.4 & 4.5 & 29 & 26.7 & $2.8<0.005$ & 3.3 \\
$\begin{array}{l}\text { Maxillary first } \\
\text { molar gingival }\end{array}$ & $\mathrm{M}$ & 29 & 28.8 & 3.4 & 29 & 33.8 & $3.7<0.05 \times 10^{4}$ & 5.4 \\
Palatal height & PH & 29 & 24.1 & 3.4 & 29 & 25.9 & $2.7<0.05$ & 2.2 \\
\hline
\end{tabular}

Pre- and postoperative palatal width and height were analyzed from cast models. Intermaxillary measurements were made. $\mathrm{C}$ denotes intercanine width, $\mathrm{M}$ denotes intermolar width, and $\mathrm{PH}$ denotes palatal depth. The palatal expansion was significantly different $(t$-test, $P<0.05)$.

provision of optimal expansion and stability [9,27-29]. However, hybrid devices might be a beneficial alternative, wherein the force is applied onto two points (tooth and bone) instead of one (tooth alone), thereby causing a parallel expansion of the palatal vault instead of just tipping the tooth. During the retention phase, the two-pointed surface maintains stability [16]. The present study confirms that a significant, sustained expansion of the maxilla and normal occlusion can be achieved using a hybrid RME device for orthognathic surgery with SARME.

In a recent systematic review, the frequency of intra- and postoperative complications following orthognathic surgery with SARME was low [30]. Based on the medical records in the current study, none of the patients experienced preoperative pain, clicking, or crepitations in the TMJ, although a few had preoperative myalgia. In a study by Østensjø et al. [31], the prevalence of TMD was reported to be low in a group of healthy adolescents. At clinical follow-up in the present study, the patients with myalgia showed improvements in their symptoms, although some showed signs of arthralgia, myalgia, and clicking sounds from the TMJ. Thus, these findings cannot confirm whether orthognathic surgery improves or worsens TMJ function; however, the frequency of TMD symptoms was low [20-22]. Most patients indicated few complications (e.g., hyposensitivity in the oral mucosa and lips) at follow-up, which supports the findings by Philips et al. [23]. These results confirm the outcomes of a previously published systematic review by Starck-Jensen and colleagues [30]; however, none of the included studies in the review assessed patient-reported outcome measures. Consequently, patient-reported outcome measures were evaluated in the present study, wherein most patients expressed satisfaction with the information received and did not regret undergoing the treatment. Furthermore, difficulties (such as biting and chewing, dental and facial appearance, speech, and self-reported pain from the TMJ) were improved following surgery. These findings are in line with those reported by Modig et al. [8], wherein the overall attitude of the patients toward SARME was reported to be positive; additionally, the researchers believed that it is important to inform the patients about the upcoming treatment method in order to improve their motivation and cooperation.

Analyses of pre- and postoperative casts revealed significant expansion of the maxilla along with normal dental occlusion following treatment, which is consistent with the literature [30,32]. In most patients, a second orthognathic surgery procedure was necessary to adjust the relationship between the jaws and to achieve a normal dental occlusion. However, some patients continued to present with malocclusion following a second surgery, indicating that some preoperative malocclusions are severe and difficult to treat. The frequency of postoperative malocclusion could indicate either relapse or suboptimal treatment results. One could speculate that the preoperative extraction of premolars to reduce the width of the maxilla could complicate maxillary expansion using the SARME technique.

Some confounders may have affected the results in this study. A control group was not included because the participants served as controls. The preoperative status in Part I was compared with the postoperative status in Part II. In addition, a previous, 3-year follow-up of both OME and SARME showed that the treatments remained stable in the patients when compared with a control group [33]. Hence, studies using a 6-month retention period, as in the present study, may report less relapse compared with those that use a 3-month stabilization period [34]. Nevertheless, further prospective, randomized studies investigating SARME treatment with the use of a hybrid RME device are warranted.

Based on the results of this study, crossbite was the most common indication for orthognathic treatment with SARME. The functional and 
self-perceived aesthetic aspects of the dentition were improved. The prevalence of postoperative TMD symptoms at follow-up was low. A low percentage of patients reported altered sensations in the lips, which is a common and expected side effect of the surgery. Significant expansion of the maxilla occurred in patients who underwent orthognathic surgery with SARME using a hybrid RME device. Most patients achieved a normal dental occlusion using the SARME technique in combination with a second orthognathic surgical procedure.

\section{Acknowledgments}

Karolinska Institute, Huddinge, Sweden is acknowledged for the support of this study as well as the patients that participated in the study.

\section{Conflict of interest}

\section{None declared.}

\section{References}

1. Behlfelt K, Linder-Aronson S, McWilliam J, Neander P, Laage-Hellman J (1990) Craniofacial morphology in children with and without enlarged tonsils. Eur J Orthod 12, 233-243.

2. Valera FC, Travitzki LV, Mattar SE, Matsumoto MA, Elias AM, Anselmo-Lima WT (2003) Muscular, functional and orthodontic changes in preschool children with enlarged adenoids and tonsils. Int J Pediatr Otorhinolaryngol 67, 761-770.

3. O'Young J, McPeek B (1987) Quality of life variables in surgical trials. J Chronic Dis 40, 513-522.

4. Burden DJ, Pine CM (1995) Self-perception of malocclusion among adolescents. Community Dent Health 12, 89-92.

5. Tamme JA, Rohnen M, Gaßling V, Ciesielski R, Fischer-Brandies H, Wiltfang J et al. (2017) Correlation of general and oral health-related quality of life in malocclusion patients treated with a combined orthodontic and maxillofacial surgical approach. J Craniomaxillofac Surg 45, 1971-1979.

6. Cunningham SJ, Hunt NP, Feinmann C (1995) Psychological aspects of orthognathic surgery: a review of the literature. Int J Adult Orhod Orthognath Surg 10, 159-172.

7. Cunningham SJ, Hunt NP, Feinmann C (1996) Perceptions of outcome following orthognathic surgery. Br J Oral Maxillofac Surg 34, 210-213.

8. Modig M, Andersson L, Wårdh I (2006) Patients' perception of improvement after orthognathic surgery: pilot study. Br J Oral Maxillofac Surg 44, 24-27.

9. Suri L, Taneja P (2008) Surgically assisted rapid palatal expansion: a literature review. Am J Orthod Dentofac Orthop 133, 290-302.

10. Zandi M, Miresmaeili A, Heidari A (2014) Short-term skeletal and dental changes following bone- borne versus tooth-borne surgically assisted rapid maxillary expansion: a randomized clinical trial study. Journal of Cranio-Maxillo-Facial Surgery 42, 1190-1195.

11. Gürler G, Akar NK, Delilbasi C, Kacar I (2018) Skeletal changes following surgically assisted rapid maxillary expansion (SARME). Eur Oral Res 52, 94-98.

12. Volk J, Kadivec M, Mušič MM, Ovsenik M (2010) Three-dimensional ultrasound diagnostics of tongue posture in children with unilateral posterior crossbite. Am J Orthod Dentofacial Orthop 138, 608-612.

13. Seeberger R, Abe-Nickler D, Hoffmann J, Kunzmann K, Zingler S (2015) One- stage toothborne distraction versus two stage bone-borne distraction in surgically assisted maxillary expansion (SARME). Oral Surg Oral Med Oral Pathol Oral Radiol 120, 693-698.

14. Kunz F, Linz C, Baunach G, Böhm H (2016) Expansion patterns in surgically assisted rapid maxillary expansion-Transpalatal distractor versus hyrax appliance. J Orofac Orthop 77,
357-365.

15. Laudemann K, Santo G, Revilla C, Harth M, Kopp S, Sader RA et al. (2011) Surgically assisted rapid maxillary expansion. J Oral Maxillofac Surg 69, 2631-2643.

16. Kayalar E, Schauseil M, Kuvat SV, Emekli U, Firatli S (2016) Comparison of tooth- borne and hybrid devices in surgically assisted rapid maxillary expansion: a randomized clinical cone-beam computed tomography study. Journal of Cranio-Maxillo-Facial Surgery 44 285-293.

17. Karagkiolidou A, Ludwig B, Pazera P, Gkantidis N, Katsaros C (2013) Survival of palatal miniscews used for orthodontic appliance anchorage: a retrospective cohort study. Am J Orthod Dentofacial Orthop 143, 767-772.

18. Wilmes B, Ngan P, Liou EJ, Franchi L, Drescher D (2014) Early class III facemask treatment with the hybrid Hyrax and Alt-RAMEC protocol. J Clin Orthod 48, 84-93.

19. Iodice G, Danzi G, Cimino R, Paduano S, Michelotti A (2013) Association between posterior crossbite, masticatory muscle pain, and disc displacement: a systematic review. Eur J Orthod 35, 737-744.

20. White CS, Dolwick MF (1992) Prevalence and variance of temporomandibular dysfunction in orthognathic surgery patients. Int J Adult Orthodon Orthognath Surg 7, 7-14

21. Wolford LM, Reiche-Fischel O, Mehra P (2003) Changes in temporomandibular joint dysfunction after orthognathic surgery. J Oral Maxillofac Surg 61, 655-660.

22. Nale JC (2014) Orthognathic surgery and the temporomandibular joint patient; a review. Oral Maxillofac Surg Clin North Am 26, 551-564.

23. Phillips $C$, Essick G (2011) Inferior alveolar nerve injury following orthognathic surgery: a review of assessment issues. J Oral Rehabil 38, 547-554.

24. Mucedero M, Covielloa A, Baccettib T, Franchib L, Cozzac P (2008) Stability factors after double-jaw surgery in class III malocclusion: a systematic review. Angle Orthod 78, 11411152 .

25. Schiffman E, Ohrbach R, Truelove E, Look J, Anderson G, Goulet JP et al. (2014) Diagnostic criteria for temporomandibular disorders (DC/TMD) for clinical and research applications: recommendations of the International RDC/TMD Consortium Network and Orofacial Pain Special Interest Group. J Oral Facial Pain Headache 28, 6-27.

26. Yekta SS, Smeets R, Stein JM, Ellrich J (2010) Assessment of trigeminal nerve functions by quantitative sensory testing in patients and healthy volunteers. J Oral Maxillofac Surg 68, 2437-2451.

27. Koudstaal MJ, Poort LJ, van der Wal KG, Wolvius EB, Prahl-Andersen B, Schulten AJ (2005) Surgically assisted rapid maxillary expansion (SARME): a review of the literature. Int J Oral Maxillofac Surg 34, 709-714.

28. Verstraaten J, Kuijpers-Jagtman AM, Mommaerts MY, Berge SJ, Nada RM, Schols JG (2010) A systematic review of the effects of bone-borne surgical assisted rapid maxillary expansion. J Craniomaxillofac Surg 38, 166-174.

29. Vilani GN, Mattos CT, de Oliveira Ruellas AC, Maia LC (2012) Long-term dental and skeletal changes in patients submitted to surgically assisted rapid maxillary expansion: a meta-analysis. Oral Surg Oral Med Oral Pathol Oral Radiol 114, 689-697.

30. Starch-Jensen T, Blaehr Lindberg Tue (2016) Transverse expansion and stability after segmental Le Fort I osteotomy versus surgically assisted rapid maxillary expansion: a systematic review. J Oral Maxillofac Res 7.

31. Ostensjo V, Moen K, Storesund T, Rosèn A (2017) Prevalence of painful temporormandibular disorders and correlation to lifestyle factors among adolescents in Norway. Pain Res Manag, May 30, doi.org/ 10.1155/2164825.

32. Lee KC, Perrino M (2017) Alar width changes due to surgically assisted rapid palatal expansion: a meta-analysis. Journal of Orthodontic Science 6, 115-122.

33. Kurt G, Altug-Ataç AT, Ataç MS, Karasu HA (2010) Stability of surgically assisted rapid maxillary expansion and orthopedic maxillary expansion after 3 years follow-up. Angle Orthod 80, 613-619.

34. Seeberger R, Gander E, Hoffmann J, Engel M. (2015) Surgical management of cross-bites in orthognathic surgery: surgically assisted rapid maxillary expansion (SARME) versus two-piece maxilla. J Craniomaxillofac Surg 43, 1109-1112. 